\title{
Variation in Electronic Spectral Parameters of Nd(III) and Er(III) with Respect to Ionic Strength of the Medium
}

\author{
SHUBHA MISHRA ${ }^{1}$, SNEHA WANKAR ${ }^{2}$, \\ PREETI VISHWAKARMA ${ }^{2}$, S.K.GHOSH ${ }^{1}$ and S.N.LIMAYE ${ }^{2}$ \\ ${ }^{1}$ School of Studies in Physics, Vikram University, Ujjain (M.P.), India \\ ${ }^{2}$ Department of Chemistry, Dr. H. S. Gour University, A Central University, \\ Sagar (M.P.) 470003, India \\ snl222@yahoo.co.in
}

Received 16 September 2014 / Accepted 25 September 2014

\begin{abstract}
The $4 f$ shells are known to have core like structure and were considered as least perturbed by changes around metal ion surroundings, however, there are evidences that the $4 f$-shells partially involved direct ligand interaction. Keeping in view this observation a systematic studies on the electronic spectral and radiative properties of [Ln(III).L] where $\mathrm{L}=$ HEDTA, EDTA and CDTA, in mediums of increasing ionic strengths $(\mu=1.0$ to 2.5$)$ of $\mathrm{NaClO}_{4}$ have been undertaken with a view to observe the effect of increased compactness of the medium on the electronic spectral parameters of $\mathrm{Ln}$ (III) ion. Variations in Oscillator strength, Judd-Ofelt parameter and radiative parameters have been examined as a function of ionic strength of medium, to explore their interdependence.
\end{abstract}

Keywords: Judd-Ofelt parameters, Transition probability, Branching ratio, Radiative life time

\section{Introduction}

The study ${ }^{1}$ of the spectral parameters of $\operatorname{Ln}(\mathrm{III})$ especially $\mathrm{Nd}(\mathrm{III})$ and $\mathrm{Er}$ (III) has been of great interest in order of the co-ordination behavior ${ }^{2}$ of $\operatorname{Ln}(\mathrm{III})$ ions. As regards to their ground term the two cations exhibit similar $\mathrm{S}$ and $\mathrm{L}$ terms with only difference in the $\mathrm{J}$ term only. The main features of the energy level structure are now well established in a wide range of host materials ${ }^{3}$. Ln(III) doped studies in different environments ${ }^{4,5}$ have been most widely reported where the tapping of suitable relaxations are being used for Laser action at room temperature. Since, Neodymium Laser can operate with high efficiency ${ }^{6}$. According to Laporte's parity selection rule it states that $f-f$ transitions are forbidden by the ED mechanism. But when the lanthanide ion is exposed under the influence of a ligand-field, the non-centrosymmetric interactions allow the mixing of electronic states of opposite parity into the $4 f$ wave functions, which however, relaxes the selection rules and the transition becomes partially allowed; it is called an induced (or forced) electric dipole transition ${ }^{7}$.

The Present work deals with the evaluation of spectral and radiative parameters of $\mathrm{Nd}(\mathrm{III})$ and $\mathrm{Er}(\mathrm{III})$ ions in HEDTA, CDTA and EDTA complexing agent environments within 
medium of varied ionic strength of $\mathrm{NaClO}_{4}$. The Judd-Ofelt theory ${ }^{8}$ has been applied to evaluate the oscillator strengths $(\mathrm{P})$ and the symmetry parameters $\left(\tau_{\lambda}\right)$ and the radiative properties of $\mathrm{Nd}(\mathrm{III})$ and $\mathrm{Er}(\mathrm{III})$ ion in different environments. The transition probabilities $\left(\mathrm{A}_{\mathrm{T}}\right)$ for intermediate transitions, relaxation timings $T_{R}$ have been examined and the variations in the evaluated parameters have been observed and discussed in light of changes in the dielectric of the medium. Judd-Ofelt theory ${ }^{9}$ defines that the susceptibility of the $4 f$ - orbital and their electronic interaction in different environment are governed by the set of three intensity parameters $\Omega \lambda(\lambda=2,4,6)$ (Tensor Operators) the susceptibility of the $4 f$ - orbital and their electronic interaction in different environment indicative of direct Ln-ligand interaction, changes in symmetry and the nature of the environment around $4 f$ - $\operatorname{Ln}(\mathrm{III})$ ion.

\section{Experimental}

Experimental solution of metal ions were prepared by direct weighing, standards metal solution were prepared by dissolving their nitrates in double distilled water or directly in required volume of the experimental sets (or oxides in minimum quantity of perchloric acids). Preparation of five sets of solution as follows (Table 1):

Table 1. Preparation of sets

\begin{tabular}{ccccc}
\hline Ln (III) & Ligand & $\mathrm{NaClO}_{4}$ & Water & Ionic strength, $\mu$ \\
\hline $0.5 \mathrm{M} \times 1 \mathrm{~mL}$ & $0.5 \mathrm{M} \times 1 \mathrm{~mL}$ & $5 \mathrm{M} \times 1.0 \mathrm{~mL}$ & $3.0 \mathrm{~mL}$ & 0.999 \\
$0.5 \mathrm{M} \times 1 \mathrm{~mL}$ & $0.5 \mathrm{M} \times 1 \mathrm{~mL}$ & $5 \mathrm{M} \times 1.5 \mathrm{~mL}$ & $2.5 \mathrm{~mL}$ & 1.416 \\
$0.5 \mathrm{M} \times 1 \mathrm{~mL}$ & $0.5 \mathrm{M} \times 1 \mathrm{~mL}$ & $5 \mathrm{M} \times 2.0 \mathrm{~mL}$ & $2.0 \mathrm{~mL}$ & 1.833 \\
$0.5 \mathrm{M} \times 1 \mathrm{~mL}$ & $0.5 \mathrm{M} \times 1 \mathrm{~mL}$ & $5 \mathrm{M} \times 2.5 \mathrm{~mL}$ & $1.5 \mathrm{~mL}$ & 2.249 \\
$0.5 \mathrm{M} \times 1 \mathrm{~mL}$ & $0.5 \mathrm{M} \times 1 \mathrm{~mL}$ & $5 \mathrm{M} \times 3.0 \mathrm{~mL}$ & $1.0 \mathrm{~mL}$ & 2.667 \\
\hline
\end{tabular}

\section{Spectral analysis}

The electronic spectra were recorded on a Systronics 2201 UV-Vis Double Beam Spectrophotometer. Cuvettes of $1 \mathrm{~cm}$ path length were used to keep reference / standard solution. The spectral recordings were for Neodymium and Erbium element has been recorded in the $\lambda$ range of $330 \mathrm{~nm}-900 \mathrm{~nm}, 350 \mathrm{~nm}-700 \mathrm{~nm}$ respectively. The spectral recordings were then used for evaluation of electronic- spectral parameters using standard equations ${ }^{10}$. Judd-Ofelt parameter and radiative parameters for the fluorescent levels of $\mathrm{Nd}(\mathrm{III})$ and $\mathrm{Er}(\mathrm{III})$ ions at different ionic strength of $\mathrm{NaClO}_{4}$ have been reported in Table 2 . (Only the hypersensitive transitions have been reported for brevity, however, the values for other assignments may be obtained from author if desired).

\section{Results and Discussion}

It is observed from the spectral recordings (Figure $1 \& 2$ ) it may be stated that the first order interaction of $\mathrm{Ln}(\mathrm{III})$ with its environments is evident from shift in spectral band shapes, their size, position and intensity. These features are infact the outcome of the [ $\mathrm{Ln}(\mathrm{III})-\mathrm{L}]$ interaction leading to variations in their microscopic features-related with oscillator strengths, symmetry parameters and the relative excitation and relaxation in the intermediate levels leading to actual photonic emission.

The oscillator strength by definition is the susceptibility of specific electronic assignment to the environment. Physically it is a measure of the strength of transition equal to the ratio of ${ }^{11}$ actual transition intensity in the aqueous phase to that of intensity radiated in ligand mediums by one electron oscillating harmonically in three directions express by three tensors proposed by Judd-Ofelt ${ }^{8,12}$. Thus, the changes in environment cause a change in the frequency of the oscillating $4 f$-electron thus reflecting changes in their response towards 
the induced electrical and magnetic dipoles imposed by encompassing radiation. These changes are often larger for the hypersensitive transitions and are significant enough to cause change in the oscillator strengths values.

A perusal of the oscillator strengths values for hypersensitive transitions for the two metal ions in three different ligand environments at five varied ionic strengths of $\mathrm{NaClO}_{4}$ (Table 2) exhibit a general increase in the oscillator strengths values with increased ionic strength. This increase in oscillator strengths is a positive evidence of increased environmental perturbation due to increased compactness of the medium. The increase in perchlorate percentage result into concomitant increase in the charge dielectric of the metal ion surrounding causing an increase in oscillator strengths values.

Table 2. Variation in the Oscillator strength values for [ $\mathrm{Ln}(\mathrm{III}) . \mathrm{L}]$ complexes with varied ionic strengths of $\mathrm{NaClO}_{4} \cdot \mathrm{Nd}(\mathrm{III})-{ }^{4} \mathrm{I}_{9 / 2}, \mathrm{Er}(\mathrm{III})-{ }^{4} \mathrm{I}_{15 / 2}$

\begin{tabular}{cccccccc}
\hline \multicolumn{2}{c}{ Assignment } & \multicolumn{2}{c}{ Energy, $\mathrm{cm}^{-1}$} & $\mathrm{Nd}(\mathrm{III})$ & \multicolumn{2}{c}{ Oscillator Strength $\left(\mathrm{P} \times 10^{-5}\right)$} \\
\hline & & Ligand & $\mu=0.99$ & $\mu=1.41$ & $\mu=1.83$ & $\mu=2.2 \mu=2.66$ \\
\hline${ }^{4} \mathrm{G}_{5 / 2}$ & \multirow{2}{*}{17300} & HEDTA & 11.1508 & 18.4273 & 18.9151 & 21.4857 & 26.4535 \\
& & EDTA & 9.94162 & 10.4693 & 11.5085 & 12.0959 & 12.9870 \\
& & CDTA & 8.65056 & 9.65057 & 10.1208 & 10.7608 & 11.6479 \\
${ }^{4} \mathrm{G}_{7 / 2}$ & \multirow{2}{*}{19100} & HEDTA & 2.16290 & 2.97486 & 3.23652 & 3.50156 & 3.61005 \\
& & EDTA & 2.01406 & 2.04994 & 2.06659 & 2.21198 & .2 .36477 \\
& & CDTA & 1.39883 & 1.92251 & 2.00174 & 2.01828 & 2.15481 \\
\hline & & & & Er(III) Oscillator Strength $\left(\mathrm{P} \times 10^{-5}\right)$ \\
\hline${ }^{2} \mathrm{H}_{11 / 2}$ & \multirow{2}{*}{19150} & HEDTA & 1.02805 & 1.04480 & 1.30667 & 1.63809 & 2.50051 \\
& & EDTA & 3.87349 & 3.96226 & 3.97400 & 4.70701 & 5.18365 \\
& & CDTA & 1.58507 & 1.81523 & 1.99109 & 2.28051 & 2.50647 \\
${ }^{4} \mathrm{~F}_{7 / 2}$ & \multirow{2}{*}{20450} & HEDTA & 0.47490 & 0.55066 & 0.63882 & 0.72511 & 0.77066 \\
& & EDTA & 1.60996 & 1.85199 & 2.14565 & 2.41590 & 2.55709 \\
& & CDTA & 1.07396 & 1.17508 & 1.26883 & 1.29704 & 1.32335 \\
\hline
\end{tabular}

Simultaneously, the Carnall's proposal ${ }^{11}$ for $\mathrm{T}_{\lambda}$ parameter is $\mathrm{T}_{\lambda} \sim<\mathrm{r}^{\mathrm{t}+1}>^{2} \cdot \mathrm{R}^{-2 \mathrm{t}-2}$

where for $\lambda=2, \mathrm{t}=1$ and 3 ; for $\lambda=4, \mathrm{t}=3$ and 5 and for $\lambda=6, \mathrm{t}=5$ and 7 expects a general trend $\mathrm{T}_{2}<\mathrm{T}_{4}<\mathrm{T}_{6}$ for $\mathrm{T}_{\lambda}$ parameters where the experimental values obtained for the present case are in agreement with the theoretical range. Amongst $\tau_{\lambda}$ parameter $\tau_{2}$ is concerned with direct metal ligand interaction whereas $\tau_{6}$ exhibit the change in symmetry associated with metal ion surroundings. The general sequence obtained for the present set of complexes (Table 3) justify the smaller values of $\tau_{2}$ due to lesser $\operatorname{Ln}(\mathrm{III})-\mathrm{L}$ interaction and greater changes in $\tau_{6}$ symmetry due to disruption of larger hydration zones associated with $\operatorname{Ln}(\mathrm{III})$ ions.

Also, variation profiles for the oscillator strengths values with respect to ionic strength have been plotted (Figures 3-6), where most the slopes have significant linear profile with $\mathrm{R}^{2}$ (regression) values very near to 0.9 and 0.99 . The ratio $\tau_{2} / \tau_{6}$ a semi empirical parameter indicative of a relative involvement of ionic charges and symmetry parameters exhibit a sequence $\mathrm{Nd}(\mathrm{III})<\mathrm{Er}$ (III) which may be on account of increased ionic potential for $\mathrm{Er}$ (III) associated with $\operatorname{Ln}(\mathrm{III})$ contraction ${ }^{13}$. The two cations undertaken for studies are similar in both $4 \mathrm{f}^{\mathrm{q}}, \mathrm{S}$ and $\mathrm{L}$ values, however, differ in their $\mathrm{J}$ terms. The observed difference in the spectral parameters is a difference expected on account of their ground term stability their concomitant ${ }^{14}$ effect on the susceptibility and population of the excitation levels in the common external field. It is interesting to observe a drift in the bonding pattern from electronic to covalent electronic from Nd(III) to Er(III). The intermediate transition levels 
and their relative responses (especially for hypersensitive transition bands) have been tabulated (Table 4) for their transition probabilities, their branching ratio and the relaxation timings. The values exhibit a gradual change with respect to ionic strength of the medium. The drifts in relaxation timings serve as an evidence for the effective influence of environment of the electronic spectral parameters.

Table 3. Variation in the Judd-Ofelt parameters $\left(\tau_{2}\right.$ and $\left.\tau_{6}\right)$ for [ $\left.\operatorname{Ln}(\mathrm{III}) . \mathrm{L}\right]$ systems with varied ionic strengths of $\mathrm{NaClO}_{4}$

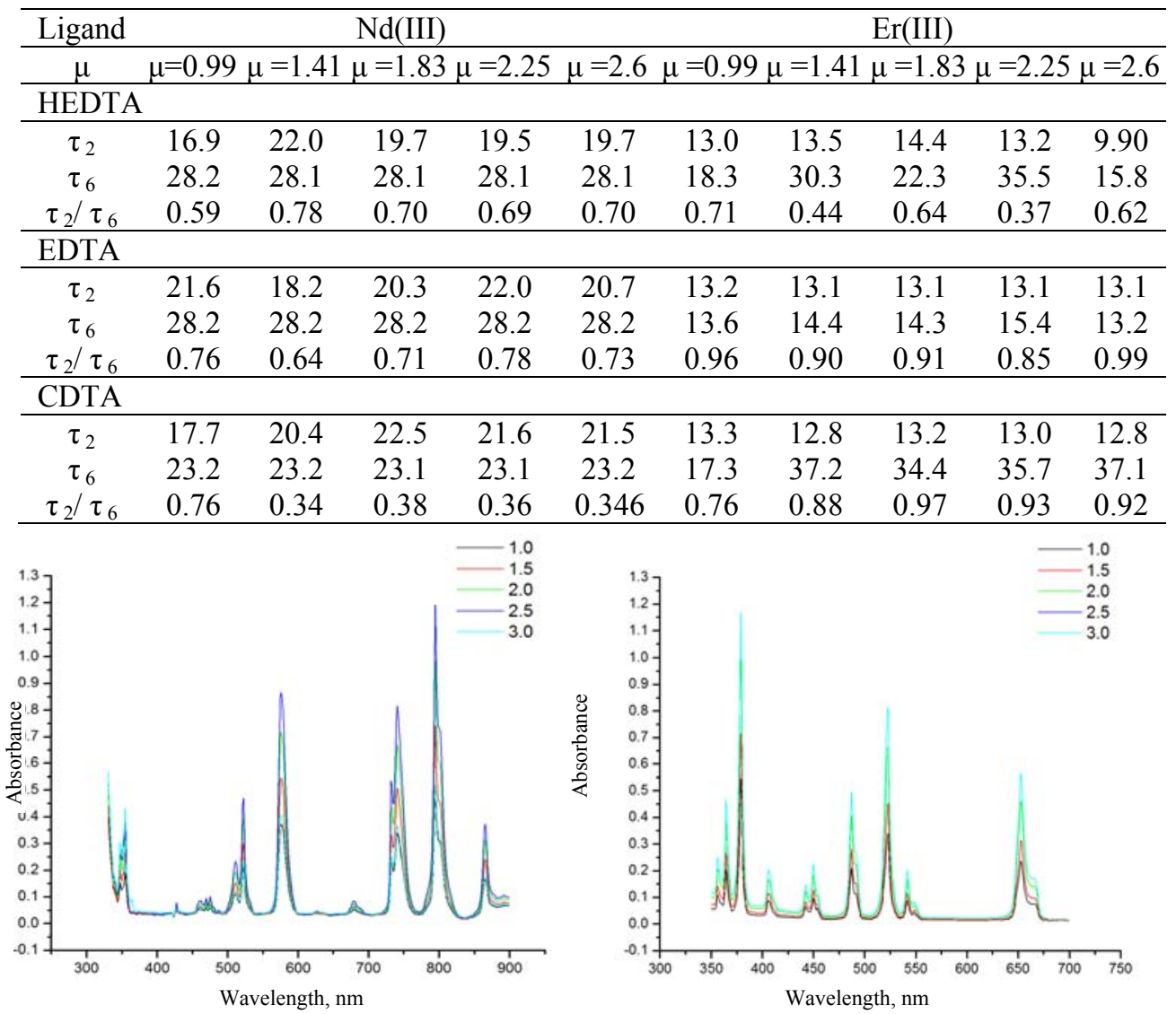

Figure 1. Absorption spectra of $\mathrm{Nd}(\mathrm{III})$

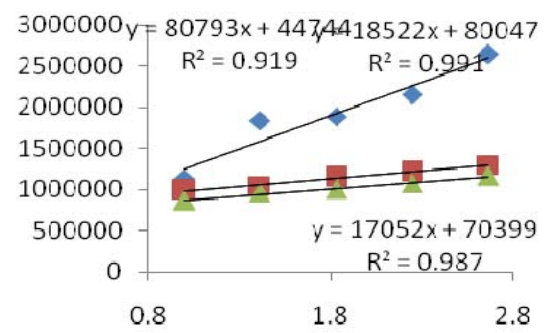

Figure 3. $\mathrm{Nd}(\mathrm{III}){ }^{4} \mathrm{G}_{5 / 2}$

Figure 2. Absorption spectra of $\operatorname{Er}(\mathrm{III})$

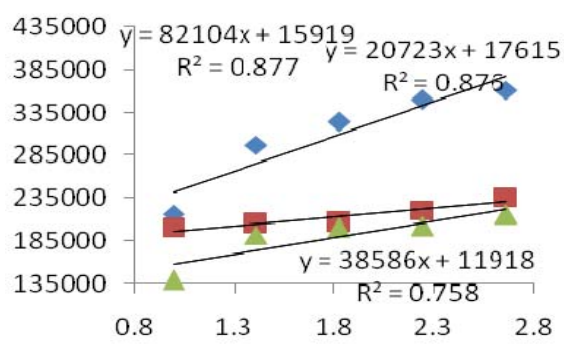

Figure 4. $\mathrm{Nd}(\mathrm{III}){ }^{4} \mathrm{G}_{7 / 2}$ 


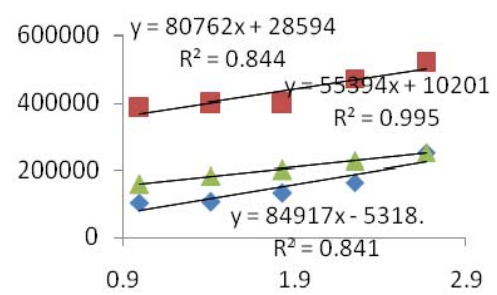

Figure 5. $\mathrm{Er}(\mathrm{III}) 2 \mathrm{H} 11 / 2$

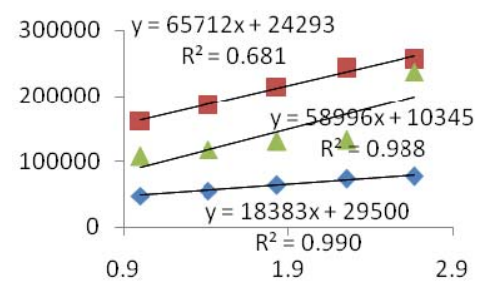

Figure 6. $\operatorname{Er}(\mathrm{III}) \quad 4 \mathrm{~F} 7 / 2$

Table 4. Variation in the Total Transition probability (AT) for [Ln(III).L] systems with varied ionic strengths of $\mathrm{NaClO}_{4}$

\begin{tabular}{cccccccc}
\hline \multicolumn{2}{r}{ Assignment } & \multicolumn{2}{c}{ Energy, $\mathrm{cm}^{-1}$} & $\mathrm{Nd}(\mathrm{III})$ & \multicolumn{3}{c}{ Total Transition probability $\left(\mathrm{A}_{\mathrm{T}}\right)$} \\
\hline & Ligand & $\mu=0.99$ & $\mu=1.41$ & $\mu=1.83$ & $\mu=2.25$ & $\mu=2.66$ \\
\hline${ }^{4} \mathrm{G}_{5 / 2}$ & 17300 & HEDTA & 15796.9 & 21177.2 & 20852.6 & 18105.7 & 20138.1 \\
& & EDTA & 20359.4 & 16753.2 & 18422 & 21307.4 & 17741.4 \\
& & CDTA & 18649.5 & 16685.3 & 22053.9 & 20529.8 & 19559.6 \\
${ }^{4} \mathrm{G}_{7 / 2}$ & 19100 & HEDTA & 12636.3 & 16731.8 & 16404.3 & 14456.7 & 16095.0 \\
& & EDTA & 16124.7 & 13374.6 & 14635.1 & 16822.8 & 14161.9 \\
& & CDTA & 14726.6 & 13207.4 & 17277.5 & 16112.5 & 15385.0 \\
\hline
\end{tabular}

\section{Conclusion}

The involvement of ionic charges and symmetry parameters exhibit a sequence $\mathrm{Nd}(\mathrm{III})<$ $\mathrm{Er}$ (III) which may be on account of increased ionic potential for $\operatorname{Er}(\mathrm{III})$ associated with Ln(III) contraction. It has been conclude that that the electronic spectral parameters of $\mathrm{Ln}$ (III) ion got perturb in different chemical environment. The $4 f$ orbital shows the change in the core like behavior because of the exposing in different environment.

\section{References}

1 Vetrone Fiorenzo, Naccache Rafik, Mahalingam Venkataramanan, Christopher G Morgan and John A Capobianc, Adv Funct Mater., 2009, 19(18), 2924-2929; DOI:10.1002/adfm.200900234

2. Elmanharawy M S, Eid A H and Abdel Kader, Czechoslovak Journal of Physics B, 1978, 28(10), 1164-1173.

2 Shriver D F, Atkins P W and Langford C H, Inorganic Chemistry, Oxford University, 1990.

3 Jayasankar C K and Ravi Kant Kumar V V, Physica B: Condensed Matter, 1996, 226, 313; DOI: 10.1016/0921-4526(96)00288-8

4 Balakrishanan R, Babu P, Jayasankar C K, Joshi A S, Speghini A and Bettinelli M, J Phys Condence Matter., 2006, 18(1), 165; DOI:10.1088/0953-8984/18/1/012

5 Devi Renuka A and Jayasankar C K, Mater Chem Phys., 1995, 42(2), 106-119; DOI:10.1016/0254-0584(95)01564-7

6 Ha"nninen P and Ha"rma" H, Lanthanide Luminescence: Photophysical, Analytical and Biological Fluoresc, 2010.

7 (a) Judd B R, Phys Rev., 1962, 127, 750-761; (b) Ofelt G S, J Chem Phys., 1962, 37(3), 511; DOI:10.1063/1.1701366

8 Jorge Henrique Santos Klier Monteiro, Italo Odone Mazali and Fernando Aparecido Sigoli, J Fluoresc, 2011, 21(6), 2237-2243; DOI:10.1007/s10895-011-0928-x

9 Agarwal A, Pal I, Sanghi S and Aggarwal M P, Optical Materials, 2009, 32, 339-344. 
10 (a) Gorller-Walrand $\mathrm{C}$ and Binnemans K, Handbook on the Physics and Chemistry of Rare-Earths, 1998, 25, 101, (b) Carnall W T, Handbook on the Physics and Chemistry of Rare-earths, 1979, 3, (c) Gschneidner K A and Jr Eyring, Handbook on the Physics and Chemistry of Rare, 1979, 24, 9; (d) Carnall W T, Fields P R and Rajnak K, J Chem Phys., 1968 , 49(10), 4424; DOI:10.1063/1.1669893

11 (a) Wong E Y, J Chem Phys., 1961, 35(2), 544; DOI:10.1063/1.1731965 (b) Wong E Y and Richman I, J Chem Phys., 1989, 36(7), 1889; DOI:10.1063/1.1701285

12 Carugo O and Castellani C B, Inorg Chimica Acta, 1992, 191(1), 115-120; DOI:10.1016/S0020-1693(00)80337-0

14. Porcher P and Caro P, J Lumin., 1980, 21, 207. 